This involved the triage of patients (including UGI Bleeds and ERCPs), coordinating with the wards and listing of patients. As the focal point of contact for ward teams, advice is given to teams about preparation of patients, as well as consenting patients on the wards ready for lists. Attending ward rounds on a daily basis to AandE, medical admissions unit and Gastroenterology wards means patients are actively listed. The effect was audited with outcomes of list utilisation, improvement patient waiting times and back-filling of lists.

Results Satisfaction Feedback from the ward staff, doctors and specialist nurses were extremely positive with the role.

ERCPS were managed more effectively with reductions in cancellations of patients that did not require them and effective triage to EUS. Also access of patients needing urgent ERCP was much easier due to the value of coordination.

UGI Bleeds Were managed more effectively which led to a reduction in the patients that required in-hours and out of hours theatre.

Interventional Endoscopy Listing in-patients for complex therapeutic interventions such as stenting and getting tertiary referrals in form other hospitals was significantly improved.

Efficiency The list utilisation improved greatly from 64 to $86 \%$ in just 8 months. The waiting times (patient scoped within $24 \mathrm{~h}$ of referral) improved by $32 \%$ for upper GI endoscopy and by $16 \%$ for sigmoidoscopy despite a substantial increase in the number of referral s (OGD increase by $13 \%$ and sigmoidoscopy by $20 \%)$.

Conclusion An in-patient liaison nurse has been pivotal for improving the quality, and efficiency, of the endoscopy service we offer to in-patients.

Disclosure of Interest None Declared.

\section{OC-055 IBD PASSPORT-DEVELOPING AN EVIDENCE-BASED INTERNET TRAVEL RESOURCE FOR INFLAMMATORY BOWEL DISEASE: A REPORT OF THE INITIAL STAGES OF IMPLEMENTATION}

K Greveson*, TC Shepherd, MI Hamilton, C Murray. Centre for Gastroenterology, Royal Free London NHS Foundation Trust, London, UK

\subsection{6/gutjnl-2014-307263.55}

Introduction Travellers with Inflammatory bowel disease (IBD) are at greater risk of travel-related morbidity ${ }^{[1]}$, with ECCO recommending expert consultation prior to travel, particularly for those on immunosuppression. ${ }^{2}$ The travel consult and patients pre-travel preparation have been found to be deficient ${ }^{1}$ Here we present the development of a dedicated IBD travel advice website to enable informed, safe travel with IBD.

Methods We conducted a literature review using Ovid databases and a search of existing online material using major internet search engines to identify existing research and resources regarding IBD and travel. Data was extracted from a recently reported prospective survey ${ }^{3}$ of 136 IBD patients which examined pre-travel preparation and experiences of travelling with IBD.

Results The database and Internet search revealed a paucity of research and resources available for IBD patients and professionals regarding travel and IBD. Our survey of 136 patients found 60\% [82/136] reported IBD affected travel, however; pre-travel medical advice was only sought by $24 \%$ [32/136]. Disease-related travel knowledge was poor with $52 \%$ of immunosuppressed patients unaware of the need to avoid live vaccines; only 53/136 (39\%) buy travel insurance covering their IBD and the majority of these (70\%) pay a premium. 91\% (124/136) would find a dedicated IBD travel website useful. As a result of this, IBDPassport ${ }^{\mathrm{TM}}$ was developed for both patients and professionals as non-commercial, IBD-specific travel resource, aimed at providing evidence-based information on all aspects of travel and IBD. The functionality of the website includes an interactive map of country specific advice including vaccinations and a 'search and refer' service for IBD professionals to refer to other IBD centres globally. Features also include practical information for travelling with IBD and specific information for the immunocompromised traveller.

Conclusion We present the first comprehensive web-based travel resource created for both IBD patients and professionals to obtain evidence-based IBD and country specific travel information. IBDPassport ${ }^{\mathrm{TM}}$ needs to be formally evaluated by patients and healthcare professionals as part of a larger study and to inform further development.

\section{REFERENCES}

1 Rahir et al. ECCO 2009

2 Soonawala et al. Inflamm Bowel Dis 2012

3 Greveson et al. A Recent Flare of Disease does not Prohibit Travel: Early Results of a Single Centre Study in Inflammatory Bowel Disease and Travel. Abstract Number: A-1908. ECCO $9^{\text {th }}$ congress Copenhagen 2014

Disclosure of Interest None Declared.

\section{OC-056 STRAIGHT TO TEST COLONOSCOPY - A VIABLE MEANS OF SHORTENING TIME TO A DEFINITIVE DIAGNOSIS}

${ }^{1} \mathrm{P}$ Andrews* ${ }^{*}{ }^{2} \mathrm{H}$ Watson, ${ }^{3} \mathrm{M}$ Mistry, ${ }^{4} \mathrm{M}$ Machesney, ${ }^{1} \mathrm{E}$ Seward. ${ }^{1}$ Endoscopy, Whipps Cross University Hospital, London, UK; ${ }^{2}$ Endoscopy, St Thomas', London, UK; ${ }^{3}$ NHS Waltham Forest CCG, London, UK; ${ }^{4}$ Colorectal Surgery, Barts Health NHS Trust, London, UK

\subsection{6/gutjnl-2014-307263.56}

Introduction Endoscopy units face an increasing demand on their ability to meet timeliness targets. One way of managing demand is to work differently - and straight to test (STT) offers this. Patients with lower gastrointestinal (LGI) symptoms are telephone triaged by a trained specialist nurse direct to the appropriate endoscopic investigation, rather than attending clinic first. Clear benefits to the patient are a reduction in time to wait until definitive (endoscopic) diagnosis, to the Trust in freeing up out-patient staff to work elsewhere, and finally to the local health economy in terms of reduction in clinic costs

Methods We followed a protocol outlined previously by the Dorset group ${ }^{1}$ Briefly, a specialist nurse assessed patients by phone and completed a symptom questionnaire. Patients were triaged according to symptoms and age; flexible sigmoidoscopy ( $<40$ yrs, anorectal symptoms only) or clinic ( $>80$ yrs and comorbidity, or major co-morbidity) or colonoscopy (everyone else). Appointments occured within 2 weeks for $2 \mathrm{WW}$ patients, or within 6 weeks for $18 \mathrm{WW}$ patients. The endoscopist was allowed to arrange further clinic review as was seen fit. A prospective database allowed capture of patient outcomes and demographic details, an estimate of financial benefit was made on the basis of standard charges for surgical $(£ 172)$ or medical $(£ 220)$ out-patient clinics.

Results 89 patients passed through the pathway in the first three months, 64\% female. Mean age 61 (range 32-88) yrs, $76 \%$ were on the $2 \mathrm{WW}$ pathway. Only $2 \%$ of the patients were triaged to flexible sigmoidoscopy, no patients required 
clinic assessment first. Mean wait on pathway for 2WW 12.4 days (range 4-20), and for 18WW 28.8 days (range 15-42), all breaches by patient choice. This represented a reduction from the normal pathway of $48 \%(2 \mathrm{WW})$ and $67 \%$ (18WW). DNA rate was low at $1 \%$ (unit average $7 \%$ ). Most common diagnoses were polyps (20\%), diverticular disease (20\%), IBD (9\%). 1 patient had colorectal cancer - a further patient was diagnosed with pancreatic cancer on CT pneumocolon following a failed colonoscopy. 13\% patients required clinic follow up. An estimated saving of $£ 14156$ was made from out-patient clinic slots that were no longer necessary, with cancellation of over seven new out-patient lists.

Conclusion These data suggest that the introduction of a novel pathway for patients with LGI symptoms can produce significant benefits to the patient in terms of time to definitive endoscopic diagnosis. A financial benefit is also clear, as is the opportunity to redeploy clinic doctors elsewhere.

\section{REFERENCE}

Wright HL. Colorectal Dis 2012;14:10

Disclosure of Interest None Declared.

\section{OC-057 IN. OUT. "NEXT PLEASE!" CAN PERSON-CENTRED CARE BE DELIVERED IN ENDOSCOPY?}

L Ferris*, S Henderson. Endoscopy Unit, Belfast City Hospital, Belfast, UK

\subsection{6/gutinl-2014-307263.57}

Introduction In. Out. "Next please!" Is this the image of endoscopy nursing? It can often be difficult to personalise care or care delivery with such a rapid turnover of patients. As registrants we have a duty to deliver safe, effective, person-centred practice (PCP). Staff at the Belfast City Hospital opted to explore this concept further.

Methods In order to understand the nature of PCP we used emancipatory practice development tools, as this is the only service improvement methodology which has the delivery of PCP as the explicit outcome. To increase awareness of "self," staff initially were invited to explore their own inherent values and beliefs, facilitated through a Values Clarification Exercise (VCE) using critical creativity ${ }^{[1]}$. The VCE helped identify key themes which formed the basis of our visioning statement. We used the 15 Steps Challenge[2] and Workplace Critical Culture Analysis Tool (WCCAT)[3] to explore the prevailing culture within the department and establish if we were indeed person-centred.

Results Integral to the PCP framework ${ }^{[1]}$ is the therapeutic relationship between service users and care providers. Working on the premise that "first impressions count," the 15 Steps Challenge was performed by key stakeholders and facilitated by junior nursing staff. Observations were made in four key areas of practice. Feedback was collated and presented to the ward manager and a subsequent action plan created, which included improvements to the reception area. In addition, to further explore the workplace culture, eight observations of practice were undertaken using the WCCAT by a representative staff group. These findings were then presented to the wider team who prioritised areas for action.

Conclusion We believe PCP should not be viewed as a one-time event, but rather a continual process embedded in everyday practice. We are confident that this premise can be extrapolated to any endoscopy unit where staff are empowered to deliver person-centred care. It is our intention to repeat the 15 Steps
Challenge and WCCAT in late 2014 to demonstrate the continual process of improvement of increasing effectiveness . ${ }^{1}$

\section{REFERENCES}

1 McCormack, B, Titchen, A. Critical creativity: melding, exploding, blending. Educational Action Research: an International Journal. 2006;14(2):239-266

2 National Health Service Institute for Innovation and Improvement. Putting Patients First - The Productive Series. Coventry: NHS Institute for Innovation and Improvement. 2012

3 McCormack B, Henderson E, Wilson V, Wright J. Making practice visible: the workplace culture critical analysis tool (WCCAT). Practice Development in Health Care 2009;8(1):28-43

4 McCormack B, McCance, T. Person-Centred Nursing Theory and Practice. Sussex, Wiley-Blackwell. 2010

5 Garbett R, McCormack B. A concept analysis of practice development. NT Research. 2002;7(2):87-100

Disclosure of Interest None Declared.

OC-058 AMBULATORY CARE ALCOHOL DETOXIFICATION (ACAD) AT WHITTINGTON HEALTH: A NEW APPROACH !

VSS Wong, R Turner*, J Pleming. Whittington Health, London, UK

10.1136/gutjnl-2014-307263.58

Introduction Alcohol-related harm costs NHS £3.5 billion a year. It has become a "top priority" for the NHS. Alcohol Concern reported 1.2 million alcohol-related admissions in 2011/12, an increase of $135 \%$ over the last 10 years, but more people are also seeking and completing treatment. Current standard treatment for those actively withdrawing from alcohol is for inpatient (IP) detoxification and concurrent assessment and intervention by an Alcohol Liaison Nurse (ALN) which usually takes, on average, 5-7 days as an IP.

Methods A care pathway was formulated for inpatients to be referred to the AC service based on strict guideline. The AC centre dedicated 2 appointments daily within working hours (Mon-Fri) for outpatient (OP) detoxification. During each session, the ALN assessed, breathalysed the patient and completed a Clinical Institute Withdrawal Scale (CIWA). The AC doctor then prescribed a daily regimen of chlordiazepoxide on a symptom-dose basis. A retrospective audit was completed of all patients referred through this pathway.

Results From Jan 13-Dec 13, 19 patients (after medically assessed) were referred for the ACAD after 3 days. 14 patients engaged with the service and completed their AD. 10 male, 4 female, aged 28-68 years. All patients were admitted via Emergency Dept; 8 presented with alcohol withdrawal, 6 with other acute problems but concurrently treated for alcohol withdrawal whilst IP. 10 had physical co-morbidities; 2 had psychiatric co-morbidities. 6 patients had a previous history of seizures. 2/14 patients consumed alcohol during the $\mathrm{AD}$ phase of their treatment (noted on breathalysing the patients). These patients were subsequently discharged from ambulatory care and referred to local alcohol services. 1 patient required readmission to hospital for physical concerns relating to diabetes, completing the remainder of the detoxification as an IP. All patients agreed to be followed up in the community and have engaged with community services. The average duration of $\mathrm{AD}$ was 5 days. In previous study we have estimated that a 7 day inpatient admission as costing $£ 2183$ vs a 3 day admission with 3 follow up ambulatory appointments costing $£ 1352$. The potential cost saving is significant once this service is widely used.

Conclusion This small innovative pilot study demonstrates that $\mathrm{ACAD}$ can be an effective and safe approach to the managing 